\title{
Microglia-conditioned medium promotes locomotor recovery and neuroprotection after rat spinal cord injury
}

\author{
May-Jywan Tsai ${ }^{1,2}$, Pei-Jou Ho ${ }^{1,3}$, Yi-Lo Lin ${ }^{1,4}$, Ming-Chao Huang ${ }^{1,2}$, Meng-Jen Lee ${ }^{1,5}$, \\ Dann-Ying Liou ${ }^{1,2}$, Wen-Cheng Huang ${ }^{1,2}$, Yu-Show Fu ${ }^{3,6^{*}}$, Henrich Cheng ${ }^{1,2,7,8,9^{*}}$ \\ ${ }^{1}$ Neural Regeneration Laboratory, Neurological Institute, Taipei Veterans General Hospital, Taipei \\ ${ }^{2}$ Center for Neural Regeneration, Neurological Institute, Taipei Veterans General Hospital, Taipei \\ ${ }^{3}$ Department of Anatomy and Cell Biology, School of Medicine, National Yang-Ming University, Taipei \\ ${ }^{4}$ Graduate Institute of Veterinary Pathobiology, College of Veterinary Medicine, National Chung Hsing University, Taichung \\ ${ }^{5}$ Institute of Biochemical Sciences and Technology, Chaoyang University of Technology, Taichung \\ ${ }^{6}$ Department of Education and Research, Taipei City Hospital, Taipei \\ ${ }^{7}$ Department of Pharmacology, School of Medicine, National Yang-Ming University, Taipei \\ ${ }^{8}$ Faculty of Medicine, School of Medicine, National Yang-Ming University, Taipei \\ ${ }^{9}$ Institute of Brain Science, School of Medicine, National Yang-Ming University, Taipei \\ Email: “ $\underline{\text { hc cheng@vghtpe.gov.tw, ysfu@ym.edu.tw }}$
}

Received 30 June 2012; revised 30 July 2012; accepted 16 August 2012

\begin{abstract}
This work examines whether microglia-conditioned medium (MCM) is beneficial in stressed spinal cord cells or tissues. MCM was separated into two fractions by $50 \mathrm{kDa}$ molecular cut-off centrifugation. MCM not only promoted survival of neuronal and oligodendroglial cells but effectively reduced LPS stimulation in spinal cord cultures. We further utilized the NYU weight-drop device to induce contusive spinal cord injury (SCI) in rats. Immediately after dropping the impactor from a height of $25 \mathrm{~mm}$ onto thoracic spinal segment, MCM was intrathecally administered. At 6 weeks post-injury, SCI rats receiving MCM > 50 kDa treatment showed significant hindlimb improvement over MCM < 50 kDa- or vehicletreated SCI rats. Consistently, more preserved nerve fibers and fewer activated microglia were found in the injured epicenter of MCM-treated SCI rats. Taken together, secreted substances, mainly $>50 \mathrm{kDa}$, of microglia was neuroprotective against spinal cord injury.
\end{abstract}

Keywords: Microglia; Neuroprotection; Spinal Cord; Anti-Inflammation

\section{INTRODUCTION}

Microglial cells are immunoreactive cells of nonneural lineage resident in the CNS and play an important role in the physiological and pathological conditions of the brain.

${ }^{*}$ Corresponding authors. These two authors contribute equally to this work.
After injury to the CNS, microglia are rapidly activated and concentrated at the sites of injury. Several studies have shown that activated microglia attribute to destructtive processes leading to secondary neuronal degeneration. However, activated microglia also expresses neuroprotective and neurotrophic activities [1-3]. A better understanding of the role of microglia and of inflammation in brain/spinal cord injuries is required to develop treatments to prevent nerve damage and improve repair.

Traumatic spinal cord injury (SCI) is an unexpected and devastating event which leads to loss of neurological function below the level of injury. SCI disrupts long projection axons and initiates a series of primary and secondary injury cascades. SCI elicits an inflammatory response that recruits macrophages to the injured spinal cord. Microglia/macrophage can become over-activated under certain circumstances and produce an excess of cytotoxic factors like superoxide, nitric oxide, and tumor necrosis factor- $\alpha$ (TNF- $\alpha$ ) [4]. These factors are strong inflammatory stimuli and contribute to neuronal loss during chronic inflammation [5]. Our previous studies demonstrated large numbers of activated macrophages present in the injury sites of repaired spinal cords after 10 days [6,7]. A portion of these recruited macrophages were likely bone marrow-derived monocytes infiltrating the injury site after 7 days [8].

A prominent role for secreted molecules in microglia has been suggested by numerous studies; however, few studies have reports or direct evidence on spinal cord. In the present work we tested whether microglia-conditioned medium (MCM) could protect spinal cord from contusive injury. Here we show that MCM was beneficial in spinal cord cultures. Furthermore, when MCM 
was intrathecally injected to spinal cord after SCI, neurological recovery was improved and spinal cord pathology was reduced.

\section{MATERIAL AND METHODS}

\subsection{Materials}

Lipopolysaccharide (LPS; Escherichia coli O111:B4) was purchased from Sigma-Aldrich (St. Louis, USA). Culture multi-wells and pipettes were obtained from Orange Scientific (Graignette, Belgium). Cultured media, fetal bovine serum (FBS) and antibiotics were purchased from Gibco (Invitrogen Corporation, USA). Other reagents were purchased from Sigma-Aldrich unless stated otherwise.

\subsection{Cell Culture}

Spinal cord neuronal/glial cultures were prepared from embryonic Sprague-Dawley (SD) rat spinal cords at gestation days 14 - 16 as described in Hung [9] and Tsai $[10,11]$. Briefly, cells were dissociated with mixtures of papain/protease/deoxyribonuclease I (0.1:0.1:0.03\%) and plated in poly-lysine coated multiwell plates $(12 \times 24$ $\mathrm{mm})$. Cells were maintained in a Dulbecco's Modified Eagle medium (DMEM, Invitrogen) supplemented with $10 \%$ FBS (Gibco BRL, Gaithersburg, $\mathrm{MD}$ ) at $37^{\circ} \mathrm{C}$ in a water-saturated atmosphere of $5 \% \mathrm{CO}_{2} / 95 \%$ air. Neurons in neuronal/glial cultures were identified by immunoreactivity to $\beta$-III tubulin (Covance, USA) and to synaptic vesicle protein 2 (SV2) (Developmental Studies Hybridoma Bank, USA). Oligodendrocytes were identified by immunoreactivity to myelin basic protein (MBP, Chemicon, USA). Microglial cells were isolated from confluent glial cell cultures as described previously [12]. Briefly, floating cells and weakly attached cells on the confluent mixed glial cell layer $[13,14]$ were isolated by gently shaking the flask for 1 - $2 \mathrm{hr}$ at $180 \mathrm{rpm}$. The resulting cell suspension was transferred to culture dish and allowed to adhere at $37^{\circ} \mathrm{C}$. Unattached cells were removed after $30 \mathrm{~min}$, microglia were isolated as strongly adhering cells. After seeding (1 day), the enriched microglial cultures were $99 \%$ positive for Iba-1 (Wako, Japan) or ED-1 (CD68, Serotec, USA) as assessed by immunostaining.

\subsection{Preparation of Microglia-Conditioned Media (MCM) and Treatment}

Medium of enriched microglia was replaced with fresh medium. Two days later, the conditioned media was collected, cleared from free-floating cells by centrifugation for $5 \mathrm{~min}$ at $1000 \times \mathrm{g}$, and sterile filtered. For fractionation of conditioned medium, MCM was further passed through an Amicon Ultracentrifugal filter with $50 \mathrm{kDa}$ cut-off (Millipore, USA) by centrifugation $3000 \mathrm{~g}$ at $4^{\circ} \mathrm{C}$. The resulted two fractions ( $>50 \mathrm{kDa}$ or $<50 \mathrm{kDa}$ ) of MCM were saved and frozen until use. For cell survival test, spinal cord neuronal/glial cultures were maintained in the presence or absence of MCM for 5 days and then fixed for immunostaining. For examining the effect of LPS stimulation, cultures were treated with MCM in the presence or absence of LPS $(1.2 \mu \mathrm{g} / \mathrm{ml})$ on the 2 nd or 3rd day after seeding. Two days later, cultured medium was collected for Griess assay of released nitrite, an indicator of nitric oxide, and the cells were fixed for immunostaining.

\subsection{Contusive Spinal Cord Injury (SCI) and Treatment}

SCI was induced in adult SD rats using the New York University weight-drop device as described previously [15]. Briefly, adult female SD rats $(250 \pm 20$ g) were anesthetized with isoflurane and a laminectomy was performed at thoracic ( $\mathrm{T}$ ) spinal cords. A contusive injury at rat $\mathrm{T} 9$ - 10 was induced by dropping a $10 \mathrm{~g}$ rod from a height of $25 \mathrm{~mm}$. Within 30 min after contusive injury, the rats received medium or MCM injection. A $5 \mu \mathrm{l} \mathrm{Ex-}$ mire microsyringe with a 31-gauge needle was positioned near the midline of the cords, $2 \mathrm{~mm}$ rostral and caudal to the contusive center. Twenty microliters of MCM (5 $\mu \mathrm{l}$ each; 4 injections) was intrathecally administered to the thoracic spinal cord $(\sim 1 \mathrm{~mm}$ ventral to the dura surface) within $30 \mathrm{~min}$ after eliciting contusive injury. Following injury and treatment, the incision was closed and sutured. Each rat was then returned to its cage. To avoid urinary tract infections, manual emptying of the urinary bladder was carried out twice daily. All surgical interventions and animal care were performed in accordance with the Laboratory Animal Welfare Act, the Guide for the Care and Use of Laboratory Animals, National Institutes of Health and were approved by the Animal Committee of Taipei Veterans General Hospital, Taiwan.

\subsection{Behavioral Assessment}

Rats were allowed to recover for 6 weeks post-injury. This time course allowed for examining the effect of MCM during the initial deficit in the behavioral locomotor assay and during the plateau phase of recovery after trauma. Recovery of hindlimb stepping was assessed while subjects moved freely about an open field. The Basso, Beattie and Bresnehan (BBB) open field score was used to evaluate locomotion in terms of hindlimb functional improvement of the SCI rats as previously described [11]. In this study, behavior analyses of SCI 
rats were conducted weekly until 6 weeks (at least 6 rats per group). The behavior tests were recorded by a video camera, and two examiners were blind to each behavior evaluation group. BBB score ranges from 0 (no hindlimb movement) to 21 (normal movement- coordinated gait). At 6 weeks following SCI and treatment, the animals were sacrificed for morphological examinations.

\subsection{Nitric Oxide (NO) Assay}

NO production was assessed using the Griess reaction. The production of NO was assayed as the accumulation of nitrite in the medium using Griess reagents ( $1 \%$ sulfanilaminde/0.1\% naphthylethylene diamine dihydrochloride/2\% phosphoric acid) as described by Tsai et al. [11]. Briefly, the culture medium was mixed with Griess reagents and incubated at room temperature for $10 \mathrm{~min}$. The absorbance of the resultant products was measured at $540 \mathrm{~nm}$. Sodium nitrite $\left(\mathrm{NaNO}_{2}\right)$ was used as a standard to calculate nitrogen dioxide $\left(\mathrm{NO}_{2}^{-}\right)$concentrations.

\subsection{Immunohistochemistry}

Cultured cells were fixed in 4\% paraformaldehyde at room temperature for $30 \mathrm{~min}$. Cells were further permeabilized with $0.2 \%$ Triton X-100, blocked with $2 \%$ serum and immunostained with primary antibodies and with the respective fluorescently tagged secondary antibodies (Jackson ImmunoResearch Inc.). Primary antibodies included mouse anti-inducible nitric oxide synthase (iNOS) (BD Bioscience, USA), rabbit anti- $\beta$ III tubulin (covance, USA) and rabbit anti-MBP (Chemicon, USA). Images of cultured cells were obtained with a fluorescent microscope equipped with fluorescence optics and with a CCD camera. Animals were perfused with 4\% paraformaldehyde at 6 weeks postinjury. The spinal cords (approximately $2 \mathrm{~mm}$ length with the contused site at the center of the sample) were removed and processed for longitudinal sectioning at a thickness of $20 \mu \mathrm{m}$. Immunostaining was performed as described previously [15]. Briefly, tissue sections were incubated with anti-Neurofilament $\mathrm{L}$ (NF-L; Chemicon, Temecula, CA) or ED-1 (Serotec, USA) antibodies overnight at $4^{\circ} \mathrm{C}$. The bound antibodies were visualized using the avidin-biotin-peroxidase complex (Vectastain Elite ABC kit; Vector Laboratories Inc., CA, USA) and with appropriate chromagens.

\subsection{Statistics}

Experimental data were expressed as the mean of independent values \pm S.E.M. One-way ANOVA followed by Bonferroni's t-test was used to determine statistical differences between treatments. A statistically significant difference was accepted at $\mathrm{P}<0.05$.

\section{RESULTS}

\subsection{Increased Cell Survival and Reduced LPS Stimulation in Spinal Cord Neuronal/Glial Cell Cultures}

Enriched microglial cells were purified from mixed glial cultures. As shown in Figures 1(A) and (B), more than 99\% of cells were immunoreactive to ED-1, a microglial marker. MCM was collected from such enriched microglia. Spinal cord neuronal/glial cultures were treated with MCM or PBS, as a vehicle control, for 5 days. Cultures were then processed for immunohistochemical staining.
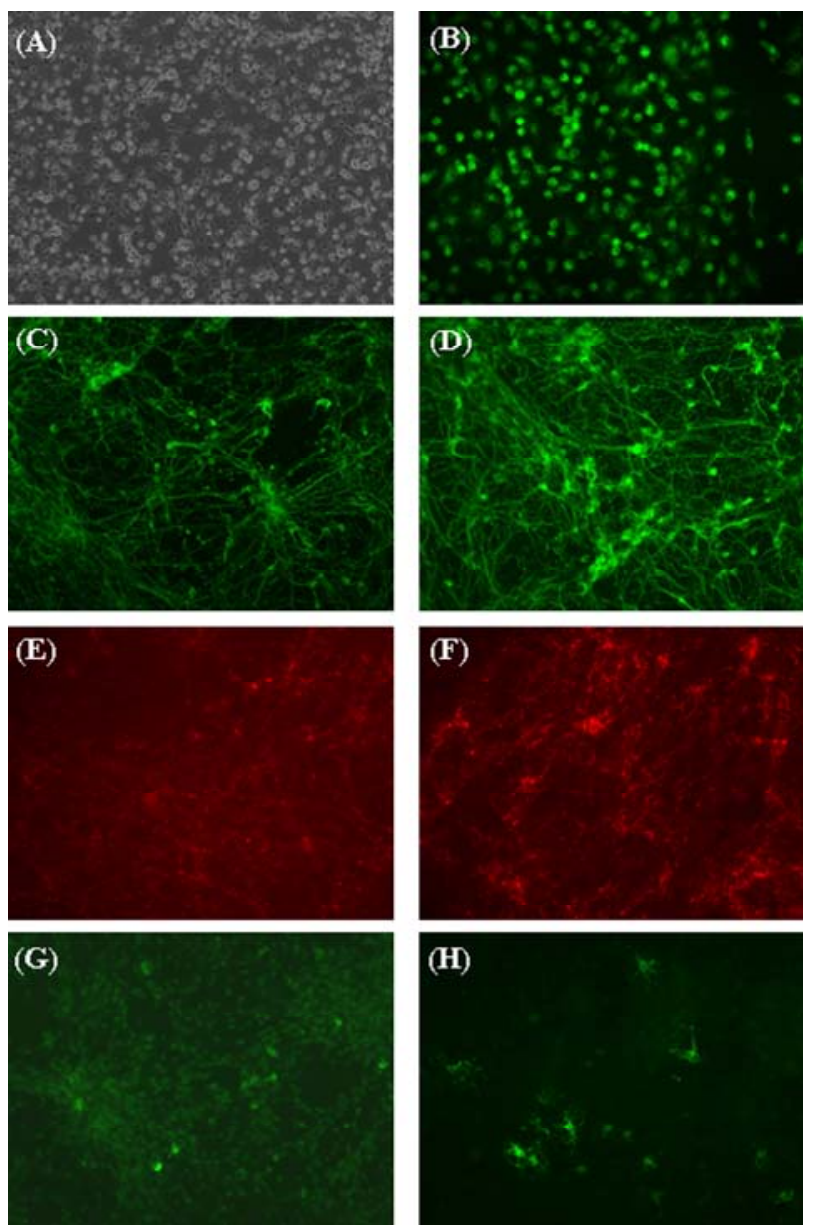

Figure 1. Analysis for effect of microglia-conditioned medium (MCM) on cell survival in spinal neuronal/glial cultures. (A), (B), (C), (E), (G): untreated control culture. (D), (F), (H): MCM-treated cultures. (A) \& (B): enriched microglial cultures (phase-contrast photo in A; ED-1-immunoreactive (IR) in B). (C) \& (D): $\beta$ III tubulin-IR cells. (E) \& (F): SV2-IR cells. (G) \& (H) myelin basic protein (MBP)-IR. Cultures were treated with MCM on the second day after cell plating. On 6 days in vitro, cell survival was analyzed using immunostaining with anti- $\beta$ III tubulin and anti-SV2, neuronal markers and with anti-MBP, an oligodendroglial marker. More $\beta$ III tubulin-IR and SV2-IR neuronal and MBP-IR oligodendroglial connections were found in MCM-treated cultures than in control cultures. 
More neurite connections of $\beta$ III were observed in MCM-treated cells (Figure 1(D)) than in control cultures (Figure 1(C)). Concurrently, the density of synaptic vesicle protein 2 (SV2)-IR was higher in MCM-treated cells than in control cells (Figures 1(E)-(F)). Interestingly, MCM treatment also increased the process of MBP $(+)$ oligodendrocytes (Figure 1(G) and (H)). Spinal cord neuronal/glial cultures were stimulated with endotoxin LPS $(1.2 \mu \mathrm{g} / \mathrm{ml})$ in the presence or absence of MCM for 2 days. Cells were then processed for iNOS immunostaining and medium were collected for nitrite detection. As shown in Figure 2, LPS induced increase of iNOS-IR cells as well as nitrite release to the medium. MCM treatment significantly reduced LPS stimulation in spinal cord cell cultures (Figures 2(A)-(D)).

\subsection{Intrathecal Administration of MCM Was Neuroprotective and Promoted Functional Recovery after SCI}

The hindlimb motor function of injured rats receiving various treatments was monitored according to the guideline of BBB Locomotor Rating Scale $[16,17]$. As shown in Figure 3, the rats receiving MCM > $50 \mathrm{kDa}$ started to show significant difference with BBB score at 2 weeks (w) after SCI, when compared to MCM < $50 \mathrm{kDa}$ group or to the control group that underwent the same procedure, but received medium treatment. Moreover, statistically significant differences in BBB scores $(\mathrm{P}<0.01)$ between MCM > 50 kDa-treated rats and MCM < 50 kDa-treated or vehicle-treated rats were found at $2 \mathrm{w}, 3 \mathrm{w}$, $4 \mathrm{w}$ and $6 \mathrm{w}$ after contusive injury and treatment. Morphological analysis of the spinal cord was conducted at 6 week post-injury. The immunohistochemical results of the injured epicenter from each group were shown in Figure 4. Contusive spinal injury increased ED-1-IR cells but reduced NF-L-IR cells. ED-1-IR cells were activated microglia/macrophage exhibiting a fully functional "amoeboid" phenotype with the ability to perform phagocytosis and generate free radicals. MCM treatment (>50 $\mathrm{kDa}$ or $<50 \mathrm{kDa}$ in Figures 4(B), (C), (E), \& (F)) reduced ED-1-IR cells and preserved NF-L-IR nerve fibers in the epicenter when compared to vehicle-treated SCI rats (Figures 4(A) \& (D)). Particularly, the injured epicenter of MCM > $50 \mathrm{kDa}$-treated rat preserved more

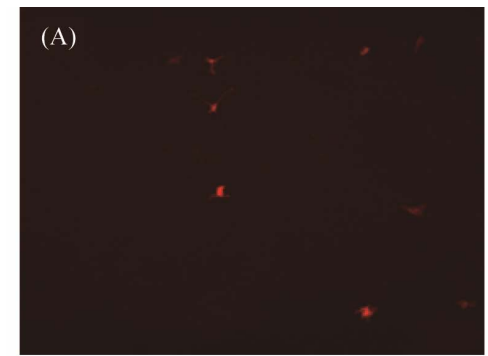

(C)

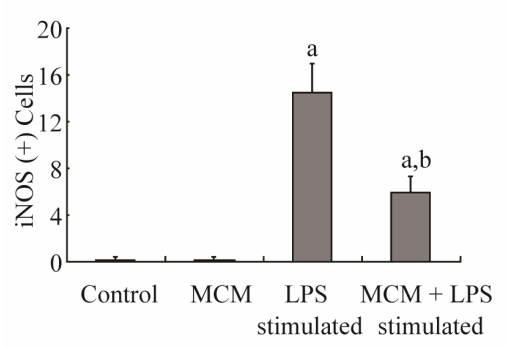

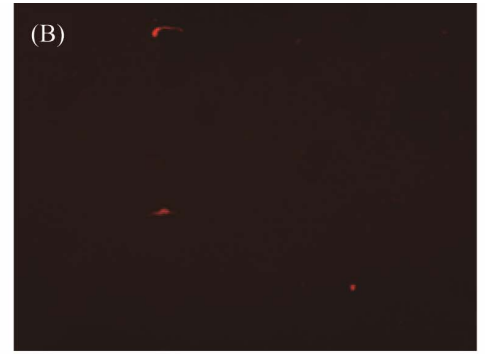

(D)

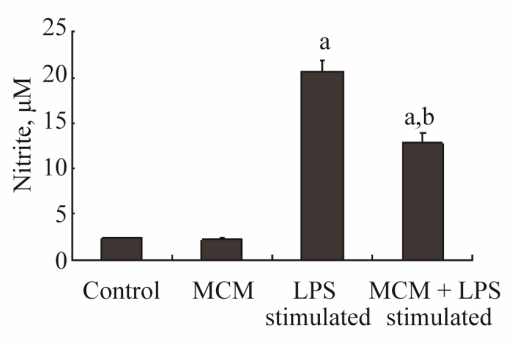

Figure 2. Effect of microglia-conditioned medium (MCM) on lipopolysaccharide (LPS) stimulation in neuronal/glial cultures of spinal cords. Cells in the images have been treated as follows: (A) LPS (1.2 $\mu \mathrm{g} / \mathrm{ml})$ for 2 days; (B) LPS $(1.2 \mu \mathrm{g} / \mathrm{ml})$ in the presence of MCM for 2 days. (A) \& (B) were cells immunoreactive (IR) to inducible NO synthase (iNOS) after treatment. Non-stimulated control or MCM-treated cells were not shown because they were not IR to iNOS. The diagram in (C) shows the number of iNOS-positive cells per 1.5 $\mathrm{mm}^{2}$ in each treatment group. LPS significantly increased iNOS $(+)$ cells $\left({ }^{\mathrm{a}} \mathrm{P}<\right.$ $0.05)$; MCM effectively reduced LPS-stimulated iNOS expression in cultures $\left({ }^{\mathrm{a}, \mathrm{b}} \mathrm{P}<0.05\right)$. The diagram in (D) shows the amount of nitrite release into the medium. LPS significantly induced nitrite release ( $\left.{ }^{\mathrm{a} P}<0.05\right)$; MCM effectively reduced LPS-stimulated nitrite release in cultures $\left({ }^{\mathrm{a}, \mathrm{b}} \mathrm{P}<0.05\right)$. ${ }^{\mathrm{a}} \mathrm{p}<0.05$, LPS vs. control in each treatment group; ${ }^{b} p<0.05$, LPS + MCM vs. LPS, by one-way ANOVA with Bonferroni's $t$-test. 


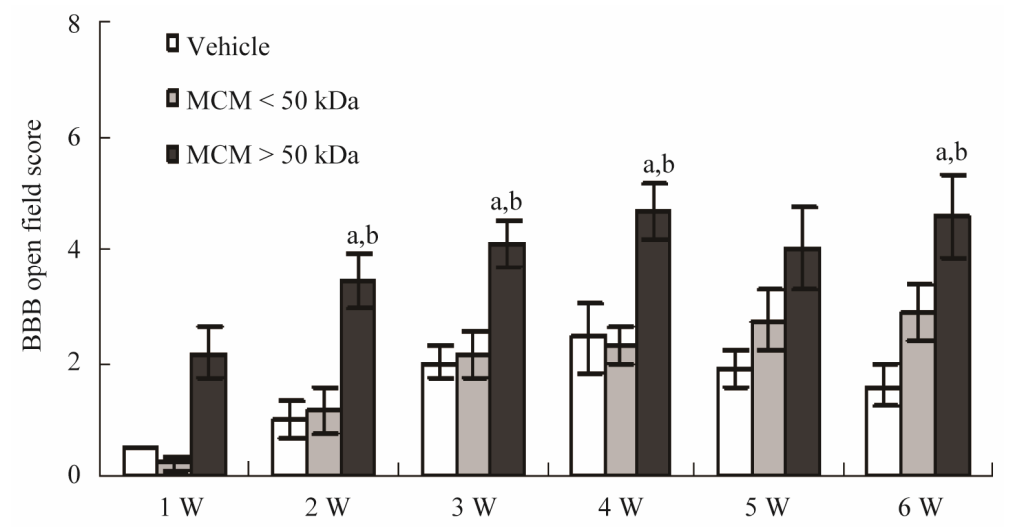

Figure 3. Time course of hindlimb locomotor recovery in rats receiving vehicleor microglia-conditioned medium (MCM) after contusive spinal injury. Time course changes of BBB scores in SCI rats. $\mathrm{N}=6$ rats for medium control; $\mathrm{N}=7$ rats each for MCM ( $>$ or $<50 \mathrm{kDa}$ )-treated group. ${ }^{\mathrm{a}} \mathrm{p}<0.01, \mathrm{MCM}>50 \mathrm{kDa}$ versus vehicle; ${ }^{\mathrm{b}} \mathrm{P}<0.01$ for $2,3,4 \mathrm{w}$ and $\mathrm{P}<0.05$ for $6 \mathrm{w}, \mathrm{MCM}>50 \mathrm{kDa}$ versus $\mathrm{MCM}<50 \mathrm{kDa}$. Statistical significance was evaluated using one-way ANOVA followed by Fishers least significant difference (LSD). Data represent the mean \pm SEM.
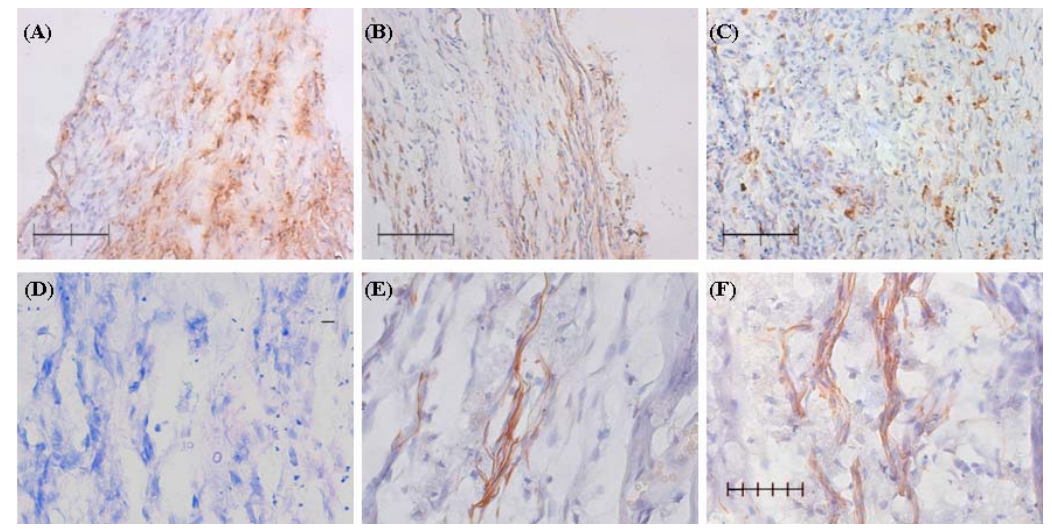

Figure 4. Microglia-conditioned medium (MCM) injection to thoracic spinal cord reduced microglial activation and preserved nerve fibers in SCI rats. ED-1- (CD68) or neurofilament (NF-L)-immunoreactivity (IR) was shown in the injury epicenter of thoracic spinal cords at 6 weeks after injury. (A), (B) \& (C) are ED-1-IR; (D), (E), (F) are NF-L-IR. Photos (A) \& (D) are from SCI rats treated with medium control; Photos (B) \& (E) are from SCI rats treated with $\mathrm{MCM}<50 \mathrm{kDa}$. Photos (C) \& (F) are from SCI rats treated with $\mathrm{MCM}>50 \mathrm{kDa}$.

\section{NF-L-IR fibers (Figure 4(F)).}

\section{DISCUSSION}

The central observation of the present study is that MCM was beneficial/anti-inflammatory to spinal cord cultures as well as to injured spinal cords in vivo. This conclusion is supported by the following evidence. First, MCM not only enhanced neuronal survival/connection, but also increased the processes of mature oligodendroglia in spinal cord cultures. Second, MCM effectively attenuated LPS stimulation in cultures. Third, intrathecal injection of MCM significantly improved functional restoration in SCI rats in vivo. Fourth, recovery in hindlimb locomotor function induced by MCM ( $>50 \mathrm{kDa}$ fraction) appeared to be correlated with the nerve fibers remained in the injured spinal cord. Fewer infiltrated ED-1 (+) microglia/ macrophages were found in the injured cord of MCMtreated SCI rats. Accordingly, the effect of MCM on functional recovery could be due to preventing neurons from insult and inflammation caused by SCI. Our results support the concept that microglia exert neuroprotective effects mainly through the release of soluble factors.

Increased neuronal and oligodendroglial connection by MCM was demonstrated by higher density of $\beta$ III-tubulinIR, SV2-IR and MBP-IR in MCM-treated cultures. Tubulin makes up microtubules. SV2-IR-synaptic vesicles 
are distributed within presynaptic terminals. The result indicated a dendritic and axonal promoting effect of MCM. MBP, a major structural protein in myelin, is responsible primarily for compaction and stabilization of the major dense line [18]. Mature oligodendroglia express MBP resulting in synthesis of a myelin membrane. MCM treatment concurrently increased oligodendroglial processes in neuron/glial cultures. We further showed that MCM conferred protection against LPS stimulation. LPS, a cell wall component of gram-negative bacteria, is a powerful immune challenge associated with an increase of numerous cytokines. Interestingly, LPS-stimulated iNOS expression and NO production were significantly attenuated by MCM. Spinal cords are prune to inflammatory stimulation. Our previous study showed that LPS at certain dosage failed to affect cortical cultures, while inflammatory mediators were highly stimulated by same dosage of LPS in spinal cord cultures [11]. The susceptibility of spinal cord to LPS stimulation may be, at least in part, attributed to the presence of immune cells and nonneuronal cells (astrocytes and microglia) [19,20].

Several lines of evidence have demonstrated that microglia could be neuroprotective after ischemia [21-25]. Yu [26] showed that grafting of activated microglia into the injured spinal cord significantly promoted the hind limb functional recovery in rats. Our results are consistent with previous reports supporting the concept that microglia exert neuroprotective effects [26-28]. Our results further showed that the released fractions, particular $>50 \mathrm{kDa}$ fraction, of microglia could improve behavioral restoration of SCI rats. Although the released factors/ compounds in MCM was not yet identified in the present studies, microglia have been reported previously to release superoxide dismutase (SOD1) [29], plasminogen [30] and neurotrophic factors (like BDNF and IL-6) [31, 32]. However, activated microglia also release excess of toxic factors (such as $\mathrm{TNF} \alpha$, IL- $1 \beta$, superoxide and nitric oxide) $[4,5]$. These factors are strong inflammatory stimuli. Fraction of MCM $>50 \mathrm{kDa}$ used in the present study had been removed off smaller molecules (such as IL1 $\beta$, TNF $\alpha$ etc) and might possess beneficial factors.

As a conclusion remark, results of the present study demonstrated a neuroprotective effect of MCM on injured spinal cord both in cultures and in vivo. These data contributed to the therapeutic use of microglia-derived products to improve repair in injured nervous system.

\section{ACKNOWLEDGEMENTS}

This work was supported by Grants V101E6-001, V100S6-001 and V99S6-001 from the Taipei Veterans General Hospital in Taiwan, by a Grant NSC 99-2628-B-010-009-MY3 from the National Science Council in Taiwan, and by a grant from the Ministry of Education (Aim for the Top University Plan).

\section{REFERENCES}

[1] Aarum, J., Sandberg, K., Haeberlein, S.L. and Persson, M.A. (2003) Migration and differentiation of neural precursor cells can be directed by microglia. Proceedings of the National Academy of Sciences USA, 100, 1598315988. doi:10.1073/pnas.2237050100

[2] Graeber, M.B., Blakemore, W.F. and Kreutzberg, G.W. (2002) Cellular pathology of the nervous system. In: Graham, D.I. and Lantos, P.L., Eds., Greenfield's Neuropathology, 7th Edition, Hodder Arnold, London, 123-191.

[3] Shaked, I., Porat, Z., Gersner, R., Kipnis, J. and Schwartz, M. (2004) Early activation of microglia as antigen-presenting cells correlates with $\mathrm{T}$ cell-mediated protection and repair of the injured central nervous system. Journal of Neuroimmunology, 146, 84-93. doi:10.1016/j.jneuroim.2003.10.049

[4] Block, M.L. and Hong, J.S. (2007) Chronic microglial activation and progressive dopaminergic neurotoxicity. Biochemical Society Transactions, 35, 1127-1132. doi:10.1042/BST0351127

[5] Minghetti, L. (2005) Role of inflammation in neurodegenerative diseases. Current Opinion in Neurology, 18, 315-321. doi:10.1097/01.wco.0000169752.54191.97

[6] Kuo, H.S., Tsai, M.J., Huang, M.C., Huang, W.C., Lee, M.J., Kuo, W.C., You, L.H., Szeto, K.C., Tsai, I.L., Chang, W.C., Chiu, C.W., Ma, H., Chak, K.F. and Cheng, H. (2007) The combination of peripheral nerve grafts and acidic fibroblast growth factor enhances arginase I and polyamine spermine expression in transected rat spinal cords. Biochemical and Biophysical Research Communications, 357, 1-7. doi:10.1016/j.bbrc.2007.02.167

[7] Kuo, H.S., Tsai, M.J., Huang, M.C., Chiu, C.W., Tsai, C.Y., Lee, M.J., Huang, W.C., Lin, Y.L., Kuo, W.C. and Cheng, H. (2011) Acid fibroblast growth factor and peripheral nerve grafts regulate Th2 cytokine expression, macrophage activation, polyamine synthesis, and neurotrophin expression in transected rat spinal cords. Journal of Neuroscience, 31, 4137-4147. doi:10.1523/JNEUROSCI.2592-10.2011

[8] Popovich, P.G. and Hickey, W.F. (2001) Bone marrow chimeric rats reveal the unique distribution of resident and recruited macrophages in the contused rat spinal cord. Journal of Neuropathology \& Experimental Neurology, 60, 676-685.

[9] Hung, H., Tsai, M.J., Wu, H.C. and Lee, E.H. (2000) Age-dependent increase in C7-1 gene expression in rat frontal cortex. Molecular Brain Research, 75, 330-336. doi:10.1016/S0169-328X(99)00325-3

[10] Tsai, M.J., Pan, H.A., Liou, D.Y., Weng, C.F., Hoffer, B.J. and Cheng, H. (2010) Adenoviral gene transfer of bone morphogenetic protein-7 enhances functional recovery after sciatic nerve injury in rats. Gene Therapy, 17, 12141224. doi:10.1038/gt.2010.72

[11] Tsai, M.J., Liao, J.F., Lin, D.Y., Huang, M.C., Liou, D.Y., Yang, H.C., Lee, H.J., Chen, Y.T., Chi, C.W., Huang, W.C. 
and Cheng, H. (2010) Silymarin protects spinal cord and cortical cells against oxidative stress and lipopolysaccharide stimulation. Neurochemistry International, 57, 867875. doi:10.1016/j.neuint.2010.09.005

[12] Tsai, M.J., Weng, C.F., Shyue, S.K., Liou, D.Y., Chen, C.H., Chiu, C.W., Yang, T.H., Pan, H.A., Liao, R.I., Kuo, H.S., Huang, M.C., Huang, W.C., Hoffer, B.J. and Cheng, H. (2007) Dual effect of adenovirus-mediated transfer of BMP7 in mixed neuron-glial cultures: Neuroprotection and cellular differentiation. Journal of Neuroscience Research, 85, 2950-2959. doi:10.1002/jnr.21395

[13] Tsai, M.J. and Lee, E.H. (1994) Differences in the disposition and toxicity of 1-methyl-4-phenylpyridinium in cultured rat and mouse astrocytes. Glia, 12, 329-335. doi:10.1002/glia.440120409

[14] Tsai, M.J. and Lee, E.H. (1998) Nitric oxide donors protect cultured rat astrocytes from 1-methyl-4-phenylpyridinium-induced toxicity. Free Radical Biology and Medicine, 24, 705-713. doi:10.1016/S0891-5849(97)00329-8

[15] Cheng, H., Wu, J.P. and Tzeng, S.F. (2002) Neuroprotection of glial cell line-derived neurotrophic factor in damaged spinal cords following contusive injury. Journal of Neuroscience Research, 69, 397-405. doi:10.1002/jnr.10303

[16] Basso, D.M., Beattie, M.S. and Bresnahan, J.C. (1995) A sensitive and reliable locomotor rating scale for open field testing in rats. Journal of Neurotrauma, 12, 1-21. doi:10.1089/neu.1995.12.1

[17] Basso, D.M., Beattie, M.S. and Bresnahan, J.C. (1996) Graded histological and locomotor outcomes after spinal cord contusion using the NYU weight-drop device versus transection. Experimental Neurology, 139, 244-256. doi:10.1006/exnr.1996.0098

[18] Pedraza, L., Fidler, L., Staugaitis, S.M. and Colman, D.R. (1997) The active transport of myelin basic protein into the nucleus suggests a regulatory role in myelination. Neuron, 18, 579-589. doi:10.1016/S0896-6273(00)80299-8

[19] Hickey, W.F. (1999) Leukocyte traffic in the central nervous system: The participants and their roles. Seminars in Immunology, 11, 125-137. doi:10.1006/smim.1999.0168

[20] Popovich, P.G., Wei, P. and Stokes, B.T. (1997) Cellular inflammatory response after spinal cord injury in Sprague-Dawley and Lewis rats. Journal of Comparative Neurology, 377, 443-464.

doi:10.1002/(SICI)1096-9861(19970120)377:3<443::AI D-CNE10>3.0.CO;2-S

[21] Imai, F., Suzuki, H., Oda, J., Ninomiya, T., Ono, K., Sano, H. and Sawada, M. (2007) Neuroprotective effect of exogenous microglia in global brain ischemia. Journal of Cerebral Blood Flow \& Metabolism, 27, 488-500. doi:10.1038/sj.jcbfm.9600362
[22] Kim, B.J., Kim, M.J., Park, J.M., Lee, S.H., Kim, Y.J., Ryu, S., Kim, Y.H. and Yoon, B.W. (2009) Reduced neurogenesis after suppressed inflammation by minocycline in transient cerebral ischemia in rat. Journal of the Neurological Sciences, 279, 70-75. doi:10.1016/j.jns.2008.12.025

[23] Lalancette-Hebert, M., Gowing, G., Simard, A., Weng, Y.C. and Kriz, J. (2007) Selective ablation of proliferating microglial cells exacerbates ischemic injury in the brain. Journal of Neuroscience, 27, 2596-2605. doi:10.1523/JNEUROSCI.5360-06.2007

[24] Neumann, J., Gunzer, M., Gutzeit, H.O., Ullrich, O., Reymann, K.G. and Dinkel, K. (2006) Microglia provide neuroprotection after ischemia. FASEB Journal, 20, 714716.

[25] Yanagisawa, D., Kitamura, Y., Takata, K., Hide, I., Nakata, Y. and Taniguchi, T. (2008) Possible involvement of P2X7 receptor activation in microglial neuroprotection against focal cerebral ischemia in rats. Biological and Pharmaceutical Bulletin, 31, 1121-1130. doi:10.1248/bpb.31.1121

[26] Yu, T.B., Cheng, Y.S., Zhao, P., Kou, D.W., Sun, K., Chen, B.H. and Wang, A.M. (2009) Immune therapy with cultured microglia grafting into the injured spinal cord promoting the recovery of rat's hind limb motor function. Chinese Journal of Traumatology, 12, 291-295.

[27] Polazzi, E. and Contestabile, A. (2002) Reciprocal interactions between microglia and neurons: from survival to neuropathology. Reviews in the Neurosciences, 13, 221242. doi:10.1515/REVNEURO.2002.13.3.221

[28] Whitney, N.P., Eidem, T.M., Peng, H., Huang, Y. and Zheng, J.C. (2009) Inflammation mediates varying effects in neurogenesis: Relevance to the pathogenesis of brain injury and neurodegenerative disorders. Journal of $\mathrm{Neu}$ rochemistry, 108, 1343-1359. doi:10.1111/j.1471-4159.2009.05886.x

[29] Polazzi, E., Mengoni, I., Caprini, M., Pena-Altamira, E., Kurtys, E. and Monti, B. (2012) Copper-Zinc Superoxide Dismutase (SOD1) is released by microglial cells and confers neuroprotection against 6-OHDA neurotoxicity. Neurosignals, Published on Line First.

[30] Nakajima, K. and Kohsaka, S. (1994) Neurotrophic effects of plasminogen. Seikagaku, 66, 533-538.

[31] Hide, I., Tanaka, M., Inoue, A., Nakajima, K., Kohsaka, S., Inoue, K. and Nakata, Y. (2000) Extracellular ATP triggers tumor necrosis factor-alpha release from rat microglia. Journal of Neurochemistry, 75, 965-972. doi:10.1046/j.1471-4159.2000.0750965.X

[32] Nakajima, K., Honda, S., Tohyama, Y., Imai, Y., Kohsaka, S. and Kurihara, T. (2001) Neurotrophin secretion from cultured microglia. Journal of Neuroscience Research, 65, 322-331. doi:10.1002/jnr.1157 